\title{
Radiale Verteilungsfunktion eines klassischen Elektronengases
}

\author{
S. Koвe und K. Voss \\ Institut für Theoretische Physik der Technischen Universität Dresden \\ (Z. Naturforschg. 22 a, 1866-1870 [1967] ; eingegangen am 19. Juli 1967)
}

\begin{abstract}
For the classical electron gas we have calculated the equilibrium radial distribution function using a modified perturbation series in powers of the plasma parameter. With the method given in this paper the divergencies of the radial distribution function for short particle distances are overcome. The modified perturbation hierarchy of the URSELL-MAYER correlation functions now can be solved in any order of the plasma parameter. The results in the first order are good with respect to the results of other methods as calculated by CARLEY.
\end{abstract}

Bei der statistischen Behandlung klassischer Plasmen treten wegen der besonderen Form der Coulomb-Kräfte bei kleinen Teilchenabständen gewisse Schwierigkeiten auf, die die Konvergenz von Störungsreihen nach Potenzen der Wechselwirkung und die Abbruchmöglichkeiten der BBKGY-Hierarchie beeinflussen (vgl. z. B. Montgomery und TiDMAN $^{1}$ ). Diese Schwierigkeiten und Wege zu ihrer Überwindung sollen in der vorliegenden Arbeit am Beispiel des klassischen Elektronengases untersucht werden.

Das klassische Elektronengas besteht aus $N$ Teilchen der Masse $m$ und der Ladung $e$ in einem gleichmäßig verschmierten, positiv geladenen neutralisierenden Untergrund. Das Wechselwirkungspotential zweier Teilchen sei

$$
v_{i j}=e^{2} /\left|\mathfrak{r}_{i}-\mathfrak{r}_{j}\right|
$$

(elektrostatische Näherung), das System habe das Volumen $\mathcal{U}$ und die Dichte $\mathscr{N}=N / \mathcal{U}$. Zur Berechnung der Eigenschaften des Systems im thermodynamischen Gleichgewicht (Temperatur $T$ ) benötigt man die Zustandssumme oder die radiale Verteilungsfunktion ${ }^{2} g\left(\left|\mathfrak{r}_{1}-\mathfrak{r}_{2}\right|\right)$. Die Verfahren $\mathrm{zu}$ deren Bestimmung beruhen entweder auf der MAYERschen Clusterentwicklung ${ }^{3,4}$ und der Verwendung von Integralgleichungen (CHNC, PERcus-Yevick-Gleichung) ${ }^{5}$ oder benutzen die Gleichungen der BBGKY-Hierarchie im zeitunabhängigen Fall ${ }^{6,7,8}$. Um die Hierarchiegleichungen nähe-

1 D. Montgomery u. D. Tidman, Plasma Kinetic Theory, McGraw-Hill, Inc., New York 1964.

2 Vgl. z.B. R. Bessenrodt, Ann. Phys. Leipzig 19, 128 [1967].

3 R. Abe, Progr. Theor. Phys. 22, 213 [1959].

4 D. L. Bowers u. E. E. Salpeter, Phys. Rev. 119, 1180 [1960].

5 D. D. Carley, Phys. Rev. 131, 1406 [1963]. rungsweise abzuschließen, kann man sie nach dem dimensionslosen Plasmaparameter

$$
\varepsilon=\mathscr{N}^{\mathbf{i} / 2}\left(e^{2} / \boldsymbol{k} T\right)^{3 / 2}
$$

entwickeln. Mit der Annahme, daß die Paarkorrelationsfunktion $h(r) \equiv g(r)-1$ mindestens von der Ordnung $\varepsilon$ ist und die Tripelkorrelation von der Ordnung $\varepsilon^{2}$, erhält man eine sukzessiv zu lösende Störungshierarchie. In der Grenze $r \rightarrow 0$ ist diese Annahme jedoch falsch und führt zu Divergenzen in der so erhaltenen radialen Verteilungsfunktion $g(r)$.

Zur Überwindung dieser Schwierigkeiten trennt Guernsey ${ }^{6}$ das Coulomb-Potential in zwei Anteile auf und entwickelt gleichzeitig nach dem Verhältnis von Reichweite des kurzreichweitigen Anteils zu mittlerem Teilchenabstand und nach dem Verhältnis des schwachen weitreichenden Anteils zur mittleren kinetischen Energie $k T$. In beiden Fällen tritt der Plasmaparameter (1) auf. O'NeIL und Rosto$\mathrm{KER}^{7}$ behandeln die Hierarchiegleichungen für große und kleine Teilchenabstände unterschiedlich und schließen die Lösungen bei $r_{0}=\mathscr{N}^{-1 / 3}$ aneinander. Unter Vernachlässigung der Tripelkorrelation wurde von Frieman und Book ${ }^{9}$ für eine nichtdivergierende Paarkorrelationsfunktion eine Differentialgleichung abgeleitet. Diese wurde von Lamb und Burdick ${ }^{10}$ gelöst. Dabei wird die Willkür der Auftrennmethoden vermieden. LIE und ICHIKAWA ${ }^{8}$ berechnen in nächsthöherer Ordnung bezüglich $\varepsilon$ den

${ }^{6}$ R. L. Guernsey, Phys. Fluids 7, 792 [1964].

7 T. O'NeIl u. N. Rostoker, Phys. Fluids 8, 1109 [1965].

8 T. L. Lie u. Y. H. Ichikawa, Rev. Mod. Phys. 38, 680 [1966].

9 E. A. Frieman u. D. L. Book, Phys.. Fluids 6, 1700 [1963].

10 G. L. Lamb u. B. Burdick, Phys. Fluids 7, 1087 [1964]. 
Einfluß der Tripelkorrelation auf $h$, erhalten jedoch dann wieder einen bei $r \rightarrow 0$ divergierenden Korrekturterm.

In der vorliegenden Arbeit soll ein neues Verfahren zur Gewinnung von Differentialgleichungen für die Korrelationsfunktionen angegeben werden, bei dem die Divergenzen bei $r \rightarrow 0$ vermieden werden können. Dazu wird die BBGKY-Hierarchie in die Hierarchie der URSELL-MAYER-Korrelationsfunktionen umgeformt und in eine dimensionslose Form gebracht. Durch eine spezielle Wahl der sukzessiven Störungshierarchie, bei der der Parameter $\varepsilon$ teilweise noch in den Gleichungen auftritt, ergibt sich $h$ in der Form

$$
h(r)=\varepsilon h^{(1)}(r, \varepsilon)+\varepsilon^{2} h^{(2)}(r, \varepsilon)+\cdots .
$$

Da $h$ in (2) nicht in eine TAYLOR-Reihe nach $\varepsilon$ um $\varepsilon=0$ entwickelt wird, können für $h^{(1)}, h^{(2)} \ldots$ bei $r \rightarrow 0$ konvergente Ausdrücke gewonnen werden.

\section{Dimensionslose UMK-Hierarchie}

Aus der LiouviLLe-Gleichung

$$
\begin{gathered}
\hat{f}_{1 \ldots N}=\left(\sum_{i=1}^{N} l_{i}+\frac{1}{2} \sum_{i \neq j} \sum_{i j} l_{i j}\right) f_{1} \ldots N, \\
\quad \int \frac{\mathrm{d} \mathrm{l} \ldots \mathrm{d} N}{N !} f_{1} \ldots N=1 \\
l_{i} \equiv-\frac{\mathfrak{p}_{i}}{m} \frac{\partial}{\partial \mathrm{r}_{i}}, \quad l_{i j} \equiv \frac{\partial v_{i j}}{\partial \mathfrak{r}_{i}}\left(\frac{\partial}{\partial \mathfrak{p}_{i}}-\frac{\partial}{\partial \mathfrak{p}_{j}}\right)
\end{gathered}
$$

$$
\begin{aligned}
\dot{u}_{1}=l_{1} & u_{1} \\
\dot{u}_{12}=\left(l_{1}\right. & \left.+l_{2}+l_{12}\right) l_{12}\left(u_{1} u_{2}+u_{12}\right), l_{12} u_{1} u_{2} \\
& +\int \mathrm{d} 3\left[l_{13} u_{1} u_{23}+l_{23} u_{2} u_{13}+\left(l_{13}+l_{23}\right)\left(u_{3} u_{12}+u_{123}\right)\right], \\
\dot{u}_{123}=\left(l_{1}\right. & \left.+l_{2}+l_{3}+l_{12}+l_{23}+l_{13}\right) u_{123}+\left(l_{12}+l_{13}\right) u_{1} u_{23} \\
& +\left(l_{12}+l_{23}\right) u_{2} u_{13}+\left(l_{23}+l_{13}\right) u_{3} u_{12} \\
& +\int \mathrm{d} 4\left[\left(l_{24}+l_{34}\right) u_{23} u_{14}+\left(l_{14}+l_{34}\right) u_{13} u_{24}+\left(l_{14}+l_{24}\right) u_{12} u_{34}\right. \\
& \left.+l_{14} u_{1} u_{234}+l_{24} u_{2} u_{134}+l_{34} u_{3} u_{124}+\left(l_{14}+l_{24}+l_{34}\right)\left(u_{4} u_{123}+u_{1234}\right)\right] .
\end{aligned}
$$

Zwar sind die Gleichungen der UMK-Hierarchie noch komplizierter miteinander gekoppelt als die der BBGKY-Hierarchie (Abb. 1), doch kann ein

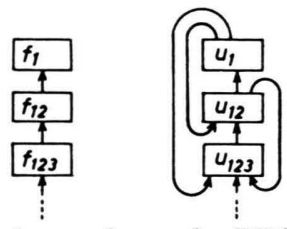

Abb. 1. Kopplungsschema der BBGKY- und der UMK-Hierarchie für die gegen Teilchenvertauschung symmetrische $N$-Teilchenverteilungsfunktion

$$
f_{1} \ldots N \equiv f\left(\mathfrak{r}_{1}, \mathfrak{p}_{1}, \ldots, \mathfrak{r}_{N}, \mathfrak{p}_{N}\right)
$$

erhält man die BBGKY-Hierarchie für die reduzierten Verteilungsfunktionen

$$
\begin{aligned}
f_{1 \ldots n}= & \int \frac{\mathrm{d}(n+1) \ldots \mathrm{d} N}{(N-n) !} f_{1 \ldots N} \\
& \int \frac{\mathrm{d} 1 \ldots \mathrm{d} n}{n !} f_{1 \ldots n}=\left(\begin{array}{l}
N \\
n
\end{array}\right)
\end{aligned}
$$

Entsprechende Hierarchiegleichungen erhält man für die URSELL-MAYER-Korrelationsfunktionen (UMK) $u_{1} \ldots n$, die über die Beziehungen

$$
\begin{aligned}
f_{1} & =u_{1} \\
f_{12} & =u_{1} u_{2}+u_{12} \\
f_{123} & =u_{1} u_{2} u_{3}+u_{1} u_{23}+u_{2} u_{13}+u_{3} u_{12}+u_{123}
\end{aligned}
$$

mit den reduzierten Verteilungsfunktionen zusammenhängen. Durch Einsetzen von (6) in (5) entsteht die Hierarchie ${ }^{11}$
Abbruch mittels Störungsentwicklung recht günstig erreicht werden.

Im Falle des hier interessierenden Gleichgewichts verschwinden die Zeitableitungen in (7), und für die $u_{i}$ kann der Ansatz

$$
u_{i}=f_{i} \sim \exp \left\{-\mathfrak{p}_{i}{ }^{2} / 2 m k T\right\}
$$

11 Die höheren Gleichungen lassen sich relativ einfach im Funktionalformalismus der klassischen Statistik mit Hilfe einer Graphenmethode erhalten; vgl. P.QuAas, K. Voss u. P. Ziesche, Acta Phys. Hung., 24 (No. 2) [1968]. 
gemacht werden. Weiterhin sollen die einzelnen Terme in (7) größenordnungsmäßig abgeschätzt werden. Dazu wird die zeitunabhängige UMKHierarchie in eine dimensionslose Form überführt. Aus den das System bestimmenden Größen $e, m, \mathscr{N}$, $k T$ lassen sich folgende charakteristische Impulse und Längen bilden:

mittlerer Impuls $\quad p_{0}=(m k T)^{1 / 2}$, LANDAU-Länge $\quad \lambda_{\mathrm{L}}=e^{2} / k T$, mittlerer Teilchenabstand $\lambda_{N}=\mathscr{N}^{-1 / 3}$,

Abschirmlänge $\quad \lambda_{0}=\left(k T / \mathscr{N} e^{2}\right)^{1 / 2}=\sqrt{4 \pi} \lambda_{\mathrm{D}}$.

Im Gegensatz zu den anderen beiden Längen ist nur die Abschirmlänge, die sich von der DebyeLänge $\lambda_{\mathrm{D}}$ um den Faktor $\sqrt{4 \pi}$ unterscheidet, sowohl von der Dichte als auch von der Wechselwirkung abhängig. Deshalb soll diese zu den folgenden Abschätzungen verwendet werden.

Impulse und Längen werden in Einheiten $p_{0}$ und $\lambda_{0}$ angegeben:

$$
\begin{gathered}
\mathfrak{p}=p_{0} \mathfrak{p}^{*}, \mathfrak{r}=\lambda_{0} \mathrm{r}^{*}, \int \mathrm{d} 3 \ldots=\left(\lambda_{0} p_{0}\right)^{3} \int \mathrm{d} 3^{*} \ldots \\
l_{1}=\left(p_{0} / m \lambda_{0}\right) l_{1}^{*}, \quad l_{12}=\left(e^{2} / \lambda_{0}^{2} p_{0}\right) l_{12}^{*} .
\end{gathered}
$$

Die mit einem Stern gekennzeichneten Größen werden dann im wesentlichen in der Größenordnung 1 liegen mit Ausnahme des Wechselwirkungsoperators $l_{12}^{*}$ bei kleinen Abständen. Um die Größenordnung der Korrelationsfunktionen $u_{1} \ldots n$ abzuschätzen, werden sie über das zur Verfügung stehende Volumen $\mathcal{U}$ integriert. Wird die Normierung (4) mit (6) verwendet und berücksichtigt, daß die $u_{1} \ldots n$ nur im Bereich der DeBye-Länge wesentlich von Null verschieden sein werden, so folgt

$$
\begin{aligned}
\text { const } \cdot N & =\int_{V} \mathrm{~d} 1 \ldots \mathrm{d} n u_{1} \ldots n \\
& \approx \int_{V} \mathrm{~d} 1 \int_{\left(\lambda_{0}^{3}\right)} \mathrm{d} 2 \ldots \mathrm{d} n u_{1} \ldots n \\
& \equiv V \lambda_{0}^{3(n-1))} p_{0}^{3 n} \bar{u}_{1} \ldots n
\end{aligned}
$$

für den Mittelwert $\bar{u}_{1 \ldots n}$. Die Korrelationsfunktionen können also durch

$$
u_{1 \ldots n}=\frac{N}{U \lambda_{0}^{3(n-1)} p_{0}^{3 n}} \bar{u}_{1 \ldots n}=\left(\mathscr{N} / p_{0}^{3}\right)^{n} \varepsilon^{n-1} u_{1 \ldots n}^{*}
$$

abgeschätzt werden, wobei $\lambda_{N} / \lambda_{0}=\varepsilon$ verwendet wurde. Nach Einsetzen von (10) und (12) in (7) mit $\dot{u}_{1} \ldots n=0$ und Multiplikation mit $m \lambda_{0} / p_{0}$ folgt

$l_{1}^{*} u_{1}^{*}+\int \mathrm{d} 2^{*} l_{12}^{*}\left(u_{1}^{*} u_{2}^{*}+\varepsilon u_{12}^{*}\right)=0$,

$\left(l_{1}^{*}+l_{2}^{*}+\varepsilon l_{12}^{*}\right) u_{12}^{*}+l_{12}^{*} u_{1}^{*} u_{2}^{*}+\int \mathrm{d} 3 *\left[l_{13}^{*} u_{1}^{*} u_{23}^{*}+l_{23}^{*} u_{2}^{*} u_{13}^{*}+\left(l_{13}^{*}+l_{23}^{*}\right)\left(u_{3}^{*} u_{12}^{*}+\varepsilon u_{123}^{*}\right)\right]=0$,

$\left(l_{1}^{*}+l_{2}^{*}+l_{3}^{*}+\varepsilon l_{12}^{*}+\varepsilon l_{23}^{*}+\varepsilon l_{13}^{*}\right) u_{123}^{*}+\left(l_{12}^{*}+l_{13}^{*}\right) u_{1}^{*} u_{23}^{*}+\left(l_{12}^{*}+l_{23}^{*}\right) u_{2}^{*} u_{13}^{*}+\left(l_{23}^{*}+l_{13}^{*}\right) u_{3}^{*} u_{12}^{*}$

$+\int \mathrm{d} 4^{*}\left[\left(l_{24}^{*}+l_{34}^{*}\right) u_{23}^{*} u_{14}^{*}+\left(l_{14}^{*}+l_{34}^{*}\right) u_{13}^{*} u_{24}^{*}+\left(l_{14}^{*}+l_{24}^{*}\right) u_{12}^{*} u_{34}^{*}\right.$

$\left.+l_{14}^{*} u_{1}^{*} u_{234}^{*}+l_{24}^{*} u_{2}^{*} u_{134}^{*}+l_{34}^{*} u_{3}^{*} u_{124}^{*}+\left(l_{14}^{*}+l_{24}^{*}+l_{34}^{*}\right)\left(u_{4}^{*} u_{123}^{*}+\varepsilon u_{1234}^{*}\right)\right]=0$.

\section{Formale Parameterentwicklung}

Da in diesem Abschnitt nur mit den dimensionslosen Größen von (13) gerechnet wird, werden dieselben hier nicht gesondert gekennzeichnet. Im Abschnitt 3 wird dann mittels (10) und (12) wieder zu dimensionsbehafteten Größen übergegangen.

Die Gln. (13) zeigen, daß die dimensionslosen UMK vom Parameter $\varepsilon$ abhängen. Sie können daher formal durch vorerst völlig willkürliche Funktionen $u_{1}^{(i)} \ldots n$ dargestellt werden:

$$
u_{1 \ldots n}=\sum_{v=0}^{\infty} \varepsilon^{v} u_{1 \ldots n}^{(n-1+v)}(\varepsilon) .
$$

Eine direkte TAYLOR-Entwicklung in (13) nach Potenzen von $\varepsilon$ würde in den Grenzfällen $r_{i j} \rightarrow 0 \mathrm{zu}$ divergenten Ausdrücken für die $u_{1} \ldots n$ führen. Das liegt daran, daß selbst bei kleinem $\varepsilon$ z. B. der Term $\varepsilon l_{12} u_{12} \sim \varepsilon / r_{12}^{2}$ bei $r \rightarrow 0$ nicht als kleine Größe angesehen werden kann. Andererseits bereiten Terme wie $\varepsilon \int \mathrm{d} 2 l_{12} u_{12}$ keine Schwierigkeiten, weil das $1 / r^{2}$ Verhalten von $l_{12}$ hier durch die räumliche Integration kompensiert wird. Nach Einsetzen von (14) in (13) werden daher die Bestimmungsgleichungen für die $u_{1 \ldots n}^{(i)}$ gerade so gewählt, daß Terme der Struktur $\varepsilon l_{12} u_{1 \ldots n}^{(n-1)}$ bereits in den Gleichungen für die jeweils niedrigste Ordnung $u_{1 \ldots n}^{(n-1)}$ enthalten sind. Dadurch tritt in diesen Gleichungen $\varepsilon$ als Parameter auf, so daß deren Lösungen $u_{1 \ldots n}^{(i)}$ wie in (14) angegeben, noch von dieser Konstante abhängen werden. So wird auch der Fehler berichtigt, der in (10) dadurch entsteht, daß das Potential $e^{2} / r$ auch für kleine $r$ mit $e^{2} / \lambda_{0} r *$ abgeschätzt wurde. 
Da man über (8) im Gleichgewicht die Funktion $u_{1}$ genau kennt und daher $u_{1}=u_{1}^{(0)}$ mit $u_{1}^{(1)}=u_{1}^{(2)}$ $=\cdots=0$ setzen kann, folgt unter Verwendung von $\int \mathrm{d} 3 l_{13} u_{3}^{(0)}=0$ die Hierarchie

$$
\begin{aligned}
& \left(l_{1}+l_{2}+\varepsilon l_{12}\right) u_{12}^{(1)}+l_{12} u_{1}^{(0)} u_{2}^{(0)}+\int \mathrm{d} 3\left(l_{13} u_{1}^{(0)} u_{23}^{(1)}+l_{23} u_{2}^{(0)} u_{13}^{(1)}\right)=0, \\
& \left(l_{1}+l_{2}+\varepsilon l_{12}\right) u_{12}^{(2)}+\int \mathrm{d} 3\left[l_{13} u_{1}^{(0)} u_{23}^{(2)}+l_{23} u_{2}^{(0)} u_{13}^{(2)}+\left(l_{13}+l_{23}\right) u_{123}^{(2)}\right]=0, \\
& \left(l_{1}+l_{2}+l_{3}+\varepsilon l_{12}+\varepsilon l_{23}+\varepsilon l_{13}\right) u_{123}^{(2)}+\left[\left(l_{12}+l_{13}\right) u_{1}^{(0)} u_{23}^{(1)}+\mathrm{zykl} .\right] \\
& \quad+\left[\int \mathrm{d} 4 l_{14}\left(u_{12}^{(1)} u_{34}^{(1)}+u_{13}^{(1)} u_{24}^{(1)}+u_{1}^{(0)} u_{234}^{(2)}\right)+(1 \leftrightarrow 2)+(1 \leftrightarrow 3)\right]=0 .
\end{aligned}
$$

Diese Hierarchie ist sukzessiv aufgebaut (Abb. 2), d. h., die Funktionen $u_{12}^{(1)}, u_{123}^{(2)}, u_{12}^{(2)}$ usw. können

nacheinander berechnet werden:

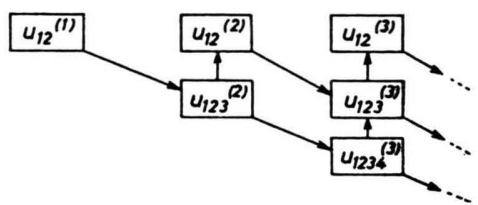

Abb. 2. Kopplungsschema der sukzessiven UMK-Hierarchie in Parameterentwicklung.
Die Auswertung von (2) erfolgt, indem

$$
h_{1 \ldots n}^{(i)} \equiv u_{1 \ldots n}^{(i)} / u_{1}^{(0)} \ldots u_{n}^{(0)}
$$

eingeführt wird. Bei homogenen und isotropen Systemen gilt $h_{12}=h_{12}\left(r_{12}\right)$ und $h_{123}\left(r_{12}, r_{23}, r_{13}\right)$. Nach Substitution der Integrale, Abspalten von $\left(\mathfrak{p}_{2}-\mathfrak{p}_{1}\right) u_{1}^{(0)} u_{2}^{(0)}$ und Anwenden von $\hat{\partial} / \partial \mathfrak{r}_{12}$ entstehen für (15a) und (15b)

$$
\begin{gathered}
\boldsymbol{D} h_{12}^{(1)}(r) \equiv \frac{\mathrm{d}^{2}}{\mathrm{~d} r^{2}} h_{12}^{(1)}+\left(\frac{2}{r}-\frac{\varepsilon}{r^{2}}\right) \frac{\mathrm{d}}{\mathrm{d} r} h_{12}^{(1)}-4 \pi h_{12}^{(1)}=0 \\
\boldsymbol{D} h_{12}^{(2)}\left(r_{12}\right)=-\frac{\partial}{\partial \mathfrak{r}_{1}} \int \mathrm{d} \mathfrak{r}_{3}\left(\frac{\partial}{\partial \mathrm{r}_{1}} \frac{1}{\left|\mathrm{r}_{1}-\mathrm{r}_{3}\right|}\right) h_{123}^{(2)}\left(r_{12}, r_{23}, r_{13}\right) .
\end{gathered}
$$

Die Gleichung für $h_{123}^{(2)}$ lautet

$$
\frac{\partial h_{123}^{(2)}}{\mathrm{d} r_{12}}-\frac{\varepsilon}{r_{12}^{2}} h_{123}^{(2)}-\frac{1}{r_{12}^{2}}\left(h_{23}^{(1)}+h_{13}^{(1)}\right)+h_{13}^{(1)} \int \mathrm{dr}_{4}\left(\frac{\partial}{\partial \mathfrak{r}_{1}} \frac{1}{r_{14}}\right) h_{24}^{(1)}+\frac{1}{2} \int \mathrm{dr}_{4}\left(\frac{\partial}{\partial r_{1}} \frac{1}{r_{14}}\right) h_{234}^{(2)}=0 .
$$

Multipliziert man (17c) mit $\mathfrak{p}_{1}\left(\mathfrak{r}_{12} / r_{12}\right) u_{1}^{(0)} u_{2}^{(0)} u_{3}^{(0)}$ und addiert anschließend alle Gleichungen, die aus (17c) durch Vertauschung von jeweils zwei Indizes bzw. zyklischen Vertauschungen entstehen, so erhält man wieder die Ausgangsgleichung (15c).

\section{Ergebnisse und Diskussion}

Zur Lösung von (17a) kann auf die Arbeiten von Lamb und Burdick 10 bzw. Lie und IChIKawa ${ }^{8}$ verwiesen werden. Das Ergebnis lautet für Systeme mit $\varepsilon \ll 1$ (über die Größenordnung von $\varepsilon$ siehe z. B. Montgomery und Tidman ${ }^{1}$ ) nach Übergang $\mathrm{zu}$ dimensionsbehafteten Größen

$h^{(1)}(r)=\left\{\begin{array}{l}-\left(\lambda_{\mathrm{L}} / r\right) \exp \left(-r / \lambda_{\mathrm{D}}\right) \\ -1+\exp \left(-\lambda_{\mathrm{L}} / r\right)\end{array} r \gtrless \sqrt{\lambda_{\mathrm{L}} \lambda_{\mathrm{D}}} / 2\right.$.

Nur für große Abstände geht (18) in den Ausdruck über, den man aus (17a) durch Weglassen des zu $\varepsilon / r^{2}$ proportionalen Terms erhält ${ }^{12}$ :

$$
h^{(1)}(r)=-\left(\lambda_{\mathrm{L}} / r\right) \exp \left(-r / \lambda_{\mathrm{D}}\right) .
$$

Der Ausdruck (19) wird jedoch für $r_{12} \approx \lambda_{\mathrm{L}}$ kleiner als -1 und hat bei $r_{12} \rightarrow 0$ eine Singularität.

12 Vgl. Montgomery u. Tidman ${ }^{1}$, Chap. 4, App. A.
Diese physikalisch unrealen Gebiete müssen bei (19) durch Abschneidevorschriften ausgeschlossen werden. Demgegenüber erfüllt (18) die geforderte Randbedingung $h^{(1)}(0)=-1$. In Tab. 1 werden (18) und die Ergebnisse von $\mathrm{O}^{\prime} \mathrm{NeIL}$ und RostokeR ${ }^{7}$ numerisch angegeben, wobei die Parameter gerade so gewählt wurden, daß ein unmittelbarer Vergleich mit den von CARLEY ${ }^{5}$ angegebenen Werten für $h_{12}$, wie sie aus anderen Rechnungen (Percus-YevickGleichung, CHNC usw.) folgen, möglich wird.

Zur Berechnung der ersten Korrektur $h_{12}^{(2)}$ aus (17b) muß zuvor $h_{123}^{(2)}$ aus (17 c) bestimmt werden. Auch hier wäre eine Vernachlässigung des Terms $\left(\varepsilon / r_{12}^{2}\right) h_{123}^{(2)}$ nicht sinnvoll, wie eine Diskussion für $r_{12} \rightarrow 0$ zeigt. Doch mit $(17 \mathrm{c})$ heben sich die zu $1 / r_{12}^{2}$ proportionalen wesentlichen Terme in der Grenze $r_{12} \rightarrow 0$ gegenseitig weg, wie aus

$$
\lim f_{123}=u_{1} u_{2} u_{3}\left(1+h_{12}+h_{23}+h_{13}+h_{123}\right)=0
$$


mit $h_{12}(0) \rightarrow-1,(6)$ und (16) folgt. Das läßt erkennen, daß (17 c) für $h_{123}^{(2)}$ einen für $r_{12}=0$ nichtdivergierenden Ausdruck liefert.

LIE und ICHIKAWA ${ }^{8}$ vernachlässigen den Term $\left(\varepsilon / r_{12}^{2}\right) h_{123}^{(2)}$, so daß nach Einsetzen eines somit nur für große Abstände gültigen $h_{123}^{(2)}$ in $(17 \mathrm{~b})$ ihr damit berechnetes $h_{12}^{(2)}$ in der Grenze $r_{12} \rightarrow 0$ eine logarith- mische Divergenz aufweist. Durch die hier entwikkelte Methode werden jedoch alle Divergenzen bei $r_{12} \rightarrow 0$ auch in höheren Ordnungen der $\varepsilon$-Entwicklung vermieden. Damit ist in Weiterführung der Arbeiten von Lie und Iснікаwa ${ }^{8}$ die Möglichkeit gegeben, die radiale Verteilungsfunktion eines klassischen Elektronengases exakter als bisher zu berechnen.

\begin{tabular}{|c|c|c|c|c|c|c|c|c|c|c|c|}
\hline \multirow[t]{2}{*}{$r / a$} & \multicolumn{2}{|c|}{$\begin{array}{c}\varepsilon=5,46 \cdot 10^{-3} \\
(\theta=20)^{13}\end{array}$} & \multicolumn{2}{|c|}{$\begin{array}{c}\varepsilon=1,55 \cdot 10^{-2} \\
(\theta=10)\end{array}$} & \multicolumn{2}{|c|}{$\begin{array}{c}\varepsilon=4,37 \cdot 10^{-2} \\
(\theta=5)\end{array}$} & \multicolumn{2}{|c|}{$\begin{array}{c}\varepsilon=9,40 \cdot 10^{-2} \\
(\theta=3)\end{array}$} & \multirow[t]{2}{*}{$r / a$} & \multicolumn{2}{|c|}{$\begin{array}{c}\varepsilon=0,489 \\
(\theta=1)\end{array}$} \\
\hline & (18) & (NR) & $(18)$ & (NR) & (18) & (NR) & $(18)$ & (NR) & & $(18)$ & $(\mathrm{NR})$ \\
\hline 0,0 & 0,000 & 0,000 & 0,000 & 0,000 & 0,000 & 0,000 & 0,000 & 0,000 & 0,0 & 0,000 & 0,000 \\
\hline 0,05 & 0,368 & 0,375 & 0,135 & 0,143 & 0,018 & 0,021 & 0,001 & 0,002 & 0,2 & 0,007 & 0,029 \\
\hline 0,1 & 0,605 & 0,618 & 0,368 & 0,388 & 0,135 & 0,157 & 0,036 & 0,049 & 0,4 & 0,083 & 0,286 \\
\hline 0,2 & 0,779 & 0,793 & 0,607 & 0,639 & 0,368 & 0,425 & 0,189 & 0,256 & 0,5 & 0,135 & 0,431 \\
\hline 0,3 & 0,852 & 0,862 & 0,716 & 0,754 & 0,513 & 0,589 & 0,329 & 0,439 & 0,6 & 0,411 & 0,555 \\
\hline 0,4 & 0,893 & 0,898 & 0,799 & 0,818 & 0,633 & 0,693 & 0,434 & 0,572 & 0,7 & 0,575 & $0,65 \overline{4}$ \\
\hline 0,5 & 0,918 & 0,921 & 0,848 & 0,859 & 0,729 & 0,762 & 0,596 & 0,667 & 0,8 & 0,687 & 0,732 \\
\hline 1,0 & 0,965 & 0,966 & 0,942 & 0,944 & 0,908 & 0,912 & 0,877 & 0,885 & 1,0 & 0,823 & 0,838 \\
\hline 2,0 & 0,989 & 0,989 & 0,983 & 0,983 & 0,979 & 0,979 & 0,977 & 0,977 & 2,0 & 0,980 & 0,980 \\
\hline
\end{tabular}

13 Es gilt $\theta \sim \varepsilon^{-2 / 3}$; vgl. CARLEY ${ }^{5}$.

Tab. 1. Radiale Verteilungsfunktion $g=1+h$ nach (18) - zum Vergleich mit den Werten von O'NEIL und $\operatorname{RosTOKER}^{7}(\mathrm{NR}) ; a=0,62 \lambda_{\mathrm{N}}$. 